\title{
EPIDEMIOLOGÍA DE LA INFECCIÓN NOSOCOMIAL EN LA UNIDAD NEONATAL HOSPITAL DE SAN JOSÉ, BOGOTÁ DC. MARZO - AGOSTO DE 2009
}

\author{
María Claudia Murcia Páez MD*, Claudia Patricia Bedoya MD**, Sandra Belén López MD**
}

\section{Resumen}

La infección nosocomial es un problema grave de salud en el país, con alta mortalidad neonatal, numerosas complicaciones, secuelas irreversibles y elevados costos y estancia hospitalaria. Se describen los factores de riesgo en el Hospital de San José (prevalencia, agentes etiológicos y mortalidad) y se comparan con la epidemiología extrainstitucional. Estudio observacional descriptivo longitudinal en los pacientes que ingresaron a la unidad neonatal procedentes de la sala de partos entre marzo y agosto de 2009. Se usó un formato de registro diario de los recién nacidos que cumplían los criterios de inclusión con seguimiento hasta el egreso. Se recolectaron 200 pacientes, 31 con sospecha clínica de infección nosocomial (53 casos) $\mathbf{2 6 . 5 \%}$. Se tuvieron en cuenta variables cualitativas y cuantitativas, analizadas como factores de riesgo y se cruzaron con infección nosocomial. Se encontró una prevalencia más baja de infección nosocomial confirmada con respecto a estadísticas de otras instituciones (5.5\%) y los gérmenes aislados fueron similares. La mayor frecuencia fue en recién nacidos a término, sugiriendo que los factores de riesgo son prevenibles, modificables y no se relacionan con los inherentes a la condición desfavorable del pretérmino y/o bajo peso al nacer. Es necesario realizar un estudio más prolongado y con mayor población para obtener resultados con significancia estadística.

Palabras clave: infección(es) nosocomial(es); recién nacidos, unidad neonatal.

Abreviaturas: IN, infección(es) nosocomial(es); RN, recién nacido(s); UN, unidad neonatal.

\section{EPIDEMIOLOGY OF NOSOCOMIAL INFECTION IN NEONATAL UNITS HOSPITAL DE SAN JOSÉ, BOGOTÁ DC. MARCH - AUGUST 2009}

\begin{abstract}
Nosocomial infection is a major health problem in our country, associated to high neonatal mortality rate, numerous complications, irreversible sequelae, high costs and longer hospital stay. The risk factors at Hospital de San José (prevalence, etiologic agents and mortality rates) are described and compared with extra institutional epidemiology. An observational longitudinal descriptive study was conducted on neonates transferred from the delivery room to the
\end{abstract}

Fecha recibido: julio 29 de 2010 Fecha aceptado: noviembre 12 de 2010

* Médica pediatra, neonatóloga, Jefe del Servicio de Pediatría, Hospital de San José. Profesora Asociada, Fundación Universitaria de Ciencias de la Salud. Bogotá DC. Colombia.
** Residente III de Pediatría, Hospital de San José. Fundación Universitaria de Ciencias de la Salud. Bogotá DC. Colombia.

*** Asesora metodológica: Esperanza Peña Torres. Directora de la División de Investigaciones Fundación Universitaria de Ciencias de la Salud. Bogotá DC. Colombia. 
neonatal unit between March and August 2009. A daily record format of the newborns that fulfilled the inclusion criteria was used and follow-up was conducted until they were discharged. Two hundred patients were collected, 31 with clinical suspicion of a nosocomial infection (53 cases) $26.5 \%$. Qualitative and quantitative variables were considered and analyzed as risk factors and crossed with nosocomial infection. The prevalence rate of confirmed nosocomial infection observed was lower than that observed in statistic records of other institutions (5.5\%) and the microorganisms isolated were similar. Frequency was greater in full term infants suggesting risk factors may be prevented, modified and are not related to the inherent unfavorable conditions of preterm/low weight at birth neonates. A more prolonged study including a larger population must be conducted in order to obtain statistically significant results.

Key words: nosocomial infection(s); NB, newborns, neonatal unit.

\section{Introducción}

La IN es un problema en las unidades neonatales ya que se asocia con aumento en la mortalidad, morbilidad, estancia hospitalaria y costos. ${ }^{1}$ Es probable que el incremento a nivel mundial en los últimos años sea consecuencia de los avances tecnológicos de la medicina y del aumento de la resistencia bacteriana de los patógenos nosocomiales en huéspedes susceptibles por su condición y patologías asociadas. ${ }^{2}$ Tiene además impactos físico, social y psicológico sobre los pacientes y sus familias, causados por eventos diferentes al motivo de su ingreso en la $\mathrm{UN} .^{3}$

En latinoamérica se ha encontrado una incidencia del 3 a $7 \%$ siendo los RN menores de 1.500 gramos los más afectados (32-87\%), con mortalidad de $51 \%$ y dos veces más probabilidades de fallecer quelos $\mathrm{RN}$ que no cursaron con IN. ${ }^{2}$ Se estima que en los Estados Unidos hay más de dos millones de IN al año entre niños y adultos y cerca de 48.600 infecciones del torrente sanguíneo asociadas con catéter. Cerca de 17.000 muertes son atribuibles a estas infecciones. ${ }^{1}$ En un estudio multicéntrico prospectivo realizado en ocho UN de Colombia en el año 2001, se encontró una incidencia de IN de 8.4\% con asociación significativa respecto al uso postnatal de esteroides, nutrición enteral y bloqueadores $\mathrm{H}_{2}{ }^{3}$

La realización de este estudio surge de la necesidad de describir y conocer las características de la IN en la UN del Hospital de San José, identificando las infecciones más frecuentes, conociendo la flora intrahospitalaria y la mortalidad, con el fin de mejorar la calidad en la atención, asertividad en el manejo antibiótico empírico, toma de medidas de acción y/o prevención, disminuyendo así la morbimortalidad de los RN. La información acerca de esta entidad es escasa a nivel local, ya que las cifras disponibles en la literatura corresponden a otros países.

\section{Materiales y métodos}

Se realizó un estudio observacional, descriptivo longitudinal en 200 pacientes nacidos en el Hospital de San José y hospitalizados enla unidad de cuidado intensivo e intermedio durante un período de seis meses, entre marzo y agosto de 2009. Los criterios de inclusión comprendieron RN del Hospital de San José hospitalizados enel área de intensivo e intermedio de la UN. Los criterios de exclusión fueron aquellos hijos de madre con corioamnionitis y procedentes de otra institución. Se registraron los datos en un formato de manera diaria así: características demográficas, procedencia, sospecha clínica de IN o confirmada por hemocultivos, localización de la infección, forma de diagnóstico, gérmenes aislados en sangre, resistencia microbiana, utilización de nutrición enteral o parenteral, ventilación mecánica, antibióticos, tubo de tórax, sonda vesical, esteroides, bloqueadores $\mathrm{H}_{2}$, catéteres (venoso periférico, central, femoral venoso y arterial, umbilical y epicutáneo), desenlace fatal y egreso. La tabulación se efectuó en Excel 2007 y el análisis de los datos se realizó con el programa estadístico Stata 10. 


\section{Resultados}

En la Tabla 1 se observan las características demográficas de la población. El tiempo promedio de estancia en cuidado intensivo fue trece días, con un máximo de 50. En el servicio de intermedios la duración de estancia máxima fue 34 días con un promedio de doce. Las intervenciones realizadas a los pacientes fueron las siguientes: procedimiento quirúrgico (cuatro pacientes, $2 \%$ ) tubo de tórax $(\mathrm{dos}, 1 \%)$ y catéter vesical (dos pacientes, $1 \%$ ). La frecuencia de utilización de bloqueadores $\mathrm{H}_{2}$ tipo ranitidina fue de $11 \%$ sin aplicación de esteroides. Se observó que 26 (12.9\%) no tuvieron nutrición enteral.

Otras intervenciones realizadas durante la hospitalización como tiempo de ventilación mecánica, nutrición parenteral y uso de catéteres se observan en la Tabla 2. El promedio de días con catéter venoso periférico fue de siete, (DS 5.27) con mínimo de uno y máximo de 21; un paciente con catéter femoral arterial durante tres días y dos con catéter femoral venoso cuyo promedio fue 22 días (DS 10.6) con mínimo de 15 días y máximo de 30; doce pacientes con catéterumbilical con promedio de 6.7 días (DS 4.04) mínimo de uno y máximo 16 días. El promedio de días con catéter epicutáneo fue de trece (DS 7,77) con mínimo de cinco y máximo de 31 días (Tabla 2).

Se diagnosticaron 31 pacientes con sospecha clínica de IN presentándose en total 53 casos (26,5\%), algunos tuvieron más de una sospecha clínica de IN distribuidos de la siguiente forma: 16 con un solo caso, ocho pacientes con dos casos y siete con tres casos.

En la Tabla 3 se observa la posible localización de la IN; en un paciente se sospechó tanto en sistema respiratorio como en gastrointestinal. El diagnóstico clínico de sepsis fue determinado por uno de los siguientes criterios: fiebre, taquicardia, taquipnea, alteración de hemograma y proteína $\mathrm{C}$ reactiva elevada en el $\mathrm{RN}$ en el cual no se identificó un sitioespecífico de infección.

\section{Tabla I. Características demográficas de la población}

\begin{tabular}{|c|c|c|c|}
\hline & & $n$ & $\%$ \\
\cline { 2 - 3 } Sexo & Femenino & 84 & 42.3 \\
& Masculino & 116 & 57.7 \\
\hline \multirow{3}{*}{ Edad gestacional } & $<28$ semanas & 5 & 19.4 \\
& 28 a 32 & 39 & 36.3 \\
& 33 a 36 & 73 & 41.8 \\
\hline Peso al nacer & $>36$ & 83 & 6 \\
& $<1.000$ gramos & 12 & 8 \\
& $1.001-1500$ & 16 & 22 \\
& $1.501-2000$ & 44 & 44 \\
\hline Edad materna & $2.001-2500$ & 40 & 6 \\
& $>2.500$ & 88 & 81.6 \\
\hline Parto & $<18$ años & 12 & 12.4 \\
\hline Procedencia & $18-35$ & 164 & 35 \\
& $>35$ & 24 & 65 \\
\hline
\end{tabular}




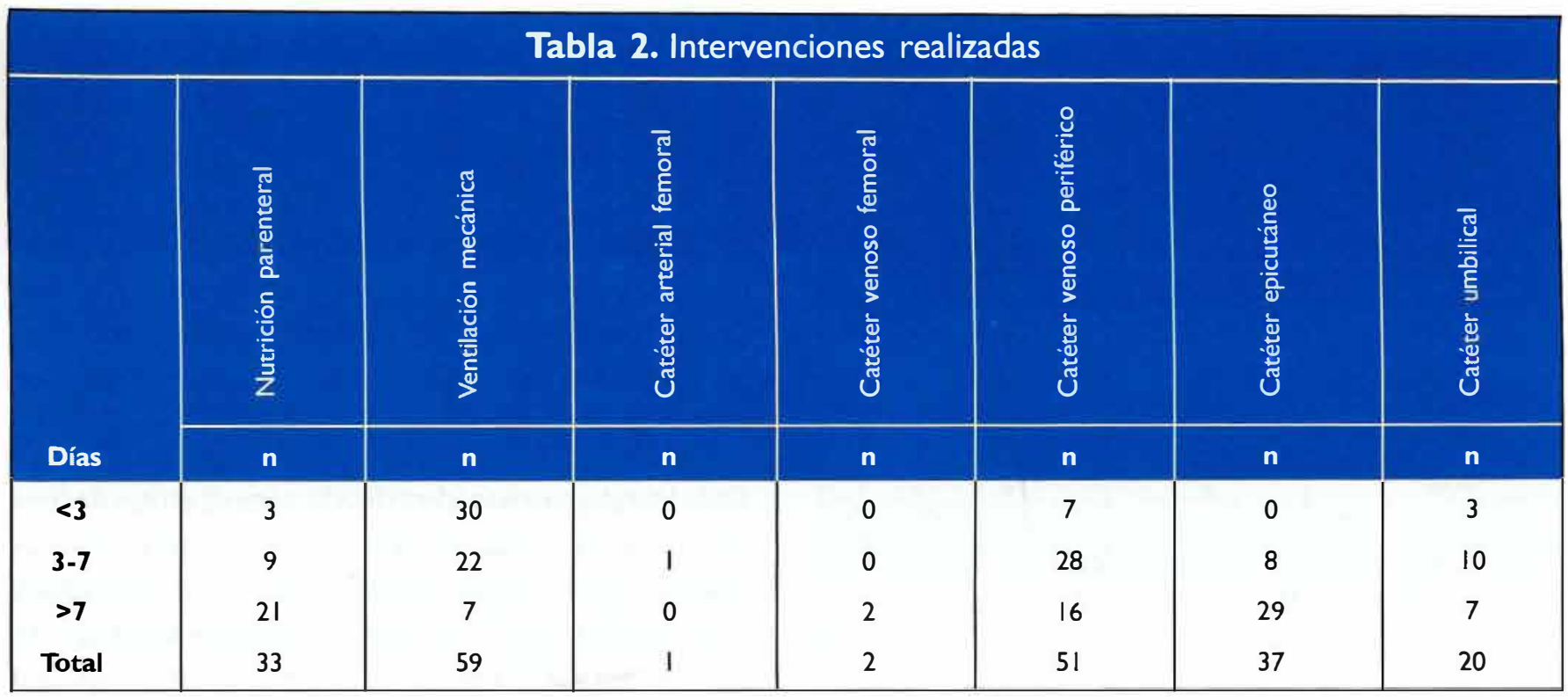

$\mathrm{n}$ : número de pacientes

Tabla 3. Localización sospechada de infección nosocomial

\begin{tabular}{|l|c|c|}
\hline & N & $\%$ \\
\hline Sistema urinario & 2 & 8 \\
Sistema gastrointestinal & 5 & 20 \\
Sistema respiratorio & 1 & 4 \\
Sepsis & 16 & 64 \\
Sistemas gastrointestinal & 1 & 4 \\
y respiratorio & & \\
Total & 25 & 100 \\
\hline
\end{tabular}

La IN confirmada por hemocultivo se observó en nueve casos y por neumonía nosocomial en dos, de las cuales una se asoció con el ventilador. La incidencia de IN corresponde al $5.5 \%$ de la población estudiada. En los hemocultivos positivos $33.3 \%$ presentaron resistencia antimicrobiana. El Staphylococcus epidermidis se observó en el $44.4 \%$ de los hemocultivos positivos (cuatro casos) siendo el germen más frecuente. En un paciente se detectó IN por varios gérmenes (Enterobacter aglomerans, Acinetobacter baumani y Serratia marcensens) en tiempos diferentes durante su hospitalización. Otro paciente desarrolló en el mismo caso de IN hemocultivo positivo y neumonía asociada con el ventilador. Durante el tiempo de seguimiento no se encontró infección fúngica. Los gérmenes documentados por hemocultivo se presentan en la Tabla 4. De los pacientes con IN confirmada, cuatro tuvieron nutrición parenteral durante más de siete días y catorce pacientes con sospecha clínica de IN tuvieron nutrición parenteral durante más de siete días.

En la Tabla 5 se muestra la relación entre IN confirmada por hemocultivo y edad gestacional. El $51 \%$ (16) de los pacientes con sospecha clínica de $\mathrm{IN}$ tuvieron ventilación mecánica, con una duración promedio de ocho días (mínima un día y máxima de 30). En seis pacientes (3\%) que recibieron antiH $\mathrm{H}_{2}$ se sospechó IN. La mortalidad fue de $9 \%$ (18 pacientes), cuyo peso al nacer fue: seis (33.3\%) menores de $1.000 \mathrm{~g}$, tres entre 1.000 y 1.500 $\mathrm{g}(16.6 \%)$, cinco entre 1.500 y $2.000 \mathrm{~g}(27.7 \%)$ y cuatro mayores de $2.500 \mathrm{~g}(22.2 \%)$. De los 31 con sospecha clínica de IN fallecieron $5(16.1 \%)$ y con IN confirmada por hemocultivo dos (Tabla 6).

Tabla 4. Gérmenes aislados en hemocultivo

\begin{tabular}{|l|c|c|}
\hline & $\mathbf{n}$ & $\%$ \\
\hline Klebsiella pneumoniae & $\mathrm{I}$ & 11.1 \\
Enterobacter aglomerans & 1 & 11.1 \\
Pseudomona stutzeri & 1 & 11.1 \\
Acinetobacter baumani & 1 & 11.1 \\
Estafilococo epidermidis & 4 & 44.4 \\
Serratia marsenses & 1 & 11.1 \\
Total & 9 & 100 \\
\hline
\end{tabular}




\begin{tabular}{|c|c|c|c|}
\hline \multicolumn{3}{|c|}{ Tabla 5. Número de pacientes con infección } \\
según edad gestacional \\
\hline $\begin{array}{c}\text { Edad gestacional } \\
\text { semanas }\end{array}$ & $\begin{array}{c}\text { Infección } \\
\text { confirmada }\end{array}$ & $\begin{array}{c}\text { Infección } \\
\text { sospechada }\end{array}$ & Total \\
\hline <28 & n & n & n \\
\hline $28-32$ & 1 & 1 & 1 \\
$33-36$ & 1 & 11 & 12 \\
$>36$ & 3 & 9 & 9 \\
TOTAL & 8 & 6 & 9 \\
\hline
\end{tabular}

$\mathrm{n}$ : número de pacientes

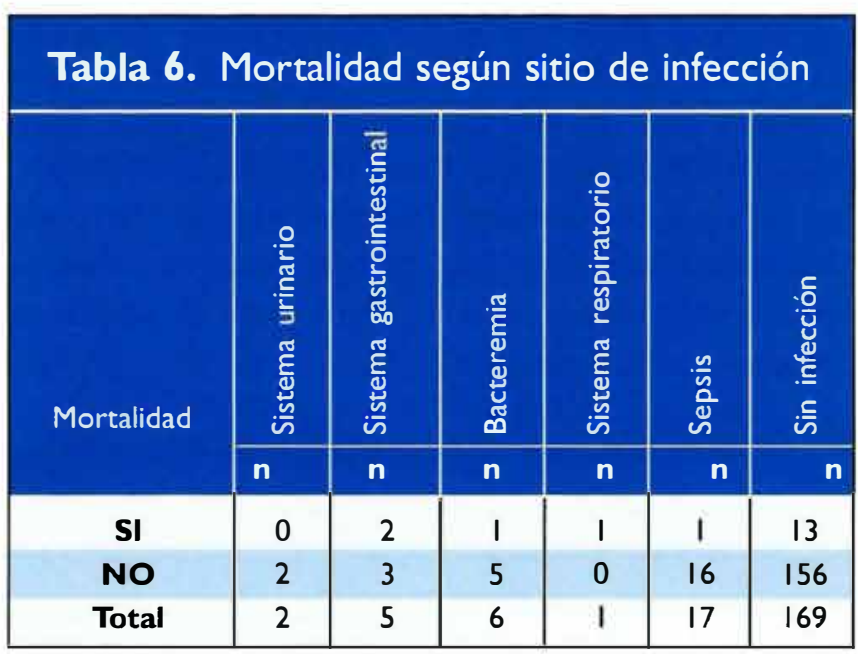

n: número de pacientes

\section{Discusión}

La incidencia de IN confirmada por hemocultivo positivo o neumonía nosocomial encontrada en el Hospital de San José de Bogotá en el período del estudio fue de $5.5 \%$, menor al encontrado en otras instituciones. ${ }^{4}$ Una de las principales razones es la población, que por ser menor podría dar un resultado sin significancia estadística. Desde el punto de vista de localización de la infección, la sepsis neonatal fue la más frecuente $(59,6 \%$ de los casos sospechosos de IN); no se logró confirmar infección urinaria ni del sistema nervioso central frente a datos encontrados en la literatura que reportan la meningitis neonatal como alto riesgo de morbilidad y mortalidad en las unidades neonatales. ${ }^{5}$
Con respecto a los microorganismos se encontró como agente etiológico principal al Staphylococcus epidermidis con $44.4 \%$ y a la Klebsiella pneumoniae con $11.1 \%$, hallazgos que coinciden con la literatura ${ }^{5} y$ con lo encontrado en los últimos tres años en la UN. La duración del estudio podría limitar el hallazgo de algunos microorganismos observados en la unidad como Enterobacter y hongos entre otros, comparados con reportes que han informado una incidencia de $I N$ por cándida entre 10 y $15 \% .^{3}$

En la literatura se han identificado como factores de riesgo: edad gestacional, bajo peso al nacer, ventilación mecánica y utilización de catéteres vasculares, esteroides y bloqueadores $\mathrm{H}_{2}{ }^{5}$ En el estudio se encontró la aplicación de nutrición parenteral durante un tiempo mayor a siete días en cuatro pacientes con IN confirmada y en catorce con sospecha clínica de IN. El 51\% de estos últimos presentó ventilación mecánicadurante un período mayor de siete días. La duración promedio de catéteres centrales femorales no superó tres días y la duración máxima ocurrió con catéter epicutáneo. No es posible concluir con estos datos y el tipo de estudio si existe o no asociación.

No se encontró uso de esteroides. El empleo de bloqueadores de receptores de histamina $\mathrm{H}_{2}$ fue de $11 \%$ en la población general, comparado con la alta asociación hallada en la investigación realizada en ocho UN de Colombia entre IN y el uso de bloqueadores $\mathrm{H}_{2}$ y esteroides ${ }^{4}$; esto está limitado por el corto tiempo de seguimiento en este estudio.

$\mathrm{Al}$ analizar la incidencia de IN según el peso al nacer se encontró que en los menores de $1.000 \mathrm{~g} \mathrm{el} 41 \%$ tuvieron sospecha de IN. Entre 1.001 y 1.500 g este porcentaje fue $31 \%$, de 1.501 a 2.000 g $28 \%$, de 2001 a $25005 \%$ y por encima de $2.500 \mathrm{~g} \mathrm{7.8 \%} \mathrm{(Tabla} \mathrm{7).} \mathrm{Estas} \mathrm{cifras} \mathrm{son}$ equivalentes a las encontradas en la literatura mundial, haciendo énfasis que en los pacientes de $2.000 \mathrm{~g}$ y más que estaban en la UN por algún evento patológico, aumentó el riesgo de infección (cardiopatía, malformaciones congénitas, asfixia, entre otros). ${ }^{6}$

La mortalidad varía según la institución, se han encontrado reportes hasta de $51 \%$ secundaria a IN.${ }^{4}$ En nuestro estudio fue de $9 \%$ en aquellos con peso al nacer por 


\begin{tabular}{|c|c|c|c|c|c|c|c|c|c|c|c|c|}
\hline \multirow[t]{2}{*}{ Infección } & \multicolumn{2}{|c|}{$<1.000 \mathrm{~g}$} & \multicolumn{2}{|c|}{$1.001-1500 \mathrm{~g}$} & \multicolumn{2}{|c|}{$1.501-2.000 \mathrm{~g}$} & \multicolumn{2}{|c|}{$2.00 \mathrm{l}-2.500 \mathrm{~g}$} & \multicolumn{2}{|c|}{$>2.500 \mathrm{~g}$} & \multicolumn{2}{|c|}{ Total } \\
\hline & $n$ & $\%$ & $n$ & $\%$ & & $\%$ & & $\%$ & & & & $\%$ \\
\hline Si & 5 & $(16.1)$ & 5 & (16.1) & & $(38,7)$ & & $(6,4)$ & 7 & (22.5) & 31 & $(100)$ \\
\hline No & 7 & $(4.1)$ & 11 & $(6,5)$ & & $(18.3)$ & 38 & $(22.4)$ & 82 & (48.5) & 169 & $(100)$ \\
\hline Total & & $(20.2)$ & 16 & $(22,6)$ & & (57) & & $(28,8)$ & 91 & (7I) & 200 & $(100)$ \\
\hline
\end{tabular}

debajo de $1.000 \mathrm{~g}$ y $16 \%$ (5 pacientes) con sospecha clínica de IN. De los pacientes con desenlace fatal el $72.2 \%$ no presentaron IN, lo cual revela que en la mayoría de los casos la causa de mortalidad se debió a otras patologías diferentes a la IN, situación que va en relación directa con la baja prevalencia de la patología.

Por último, se propone continuar con este estudio por un tiempo más prolongado y así lograr resultados con mejor significancia estadística en cuanto al número de pacientes. Además ayudaría a la prevención de la IN en la UN del hospital y a realizar un manejo aun más acertado.

\section{Conclusión}

La IN en las unidades de cuidado intensivo neonatal son unproblema de salud a nivel mundial, que va en aumento no solo por el tipo de pacientes que allí se manejan sino por la gran variedad de procedimientos invasivos necesarios que se realizan para disminuir la morbimortalidad. Los gérmenes más comunes son el Staphylococcus epidermidis causando cerca del 50\% de las infecciones, seguido por la Klebsiella pneumoniae y otros Gram negativos, todos ellos de difícil manejo. Del conocimiento de estos factores de riesgo, los gérmenes causales y el cuidadoso manejo de los RN, minimizando o acortando el tiempo de los procedimientos, depende en gran parte el control de estas patologías y la disminución de su frecuencia.

\section{Referencias}

1 Carey A, Saiman L, Polin R. Hospital-acquired infections in the NICU: epidemiology for the new millennium. Clin.Perinatol. 2008; 35(1):223-49.

2 Hernandez H, Gonzalez N, Castañeda J, Barbosa P, Saldana C, Monroy A, et al. Infecciones nosocomiales en el Instituto Nacional de Pediatria(INP 20042005). Acta Pediatr Mex. 2006; 27(6): 325-28.

3 Rojas, M, et al. Risk factors for nosocomial infections in selected neonatal intensive care units in Colombia, South America. J.Perinatol. 2005 Ago; 25(8): 537-41.

4 Mendívil C, Egüés J, Polo P, Ollaquindia P, Nuin M, Del Real C. Infección nosocomial, vigilancia y control de la infección en neonatología. An Pediatr (Barc). 2000; 23(2): 177-84.

5 Xu Y, Zhang LJ, Ge HY, Wang DH. [Clinical analysis of nosocomial infection in neonatal intensive care units]. Zhonghua Er Ke Za Zhi. 2007 Jun; 45(6):437-41.

6 Stover BH, Shulman ST, Bratcher DF, Brady MT, Levine GL, Jarvis WR. Nosocomial infection rates in US children's hospitals'neonatal and pediatric intensive care units. Am J Infect Control. 2001 Jun; 29(3):152-7. 